\title{
Estimating the Spatial Orientation of Immobilized Magnetic Nanoparticles with Parallel-Aligned Easy Axes
}

\author{
Martin Möddel $\odot,{ }^{1,2, *}$ Florian Griese $\odot,{ }^{1,2}$ Tobias Kluth $\odot,{ }^{3,4}$ and Tobias Knopp $\odot^{1,2}$ \\ ${ }^{1}$ Section for Biomedical Imaging, University Medical Center Hamburg-Eppendorf, Hamburg 20246, Germany \\ ${ }^{2}$ Institute for Biomedical Imaging, Hamburg University of Technology, Hamburg 21073, Germany \\ ${ }^{3}$ Center for Industrial Mathematics, University of Bremen, Bremen 28359, Germany \\ ${ }^{4}$ Center for Optimization and Approximation, University of Hamburg, Hamburg 20146, Germany
}

(Received 24 February 2021; revised 2 March 2021; accepted 3 September 2021; published 28 October 2021)

\begin{abstract}
Advances in micromachinery and nanotechnology, such as magnetically actuated microrobots for navigating in viscous environments, are important driving forces in medicine. Recently, it has been shown that the spatial orientation of an ensemble of immobilized nanoparticles with parallel-aligned magnetic easy axes has an effect on the magnetization response to an external dynamic magnetic field. Here, we introduce a method that allows us to estimate the spatial orientation of this axis from the magnetization response and experimentally study a potential application scenario.
\end{abstract}

DOI: 10.1103/PhysRevApplied.16.L041003

Introduction.-Magnetic nanoparticles are the subject of intense study for researchers from a wide range of disciplines [1-4]. Their key feature is that they can be detected and manipulated by static and dynamic external magnetic fields, to which they respond with Brownian and Néel rotational dynamics of their magnetization $[5,6]$. When nanoparticles are immobilized, Néel rotation dominates the magnetization dynamics. For nanoparticles with parallel alignment of the easy axes, the orientation of the easy axes with respect to the magnetic excitation field is one of the key parameters for their magnetization response [7] for large degrees of parallel alignment [8].

In this work, we use this dependency to estimate the spatial orientation of the easy axes relative to an external magnetic excitation field from the magnetization response of the nanoparticles. We approach this issue at three different levels. Theoretically, we analyze the interplay between easy-axis orientation, the external magnetic field, and the magnetic moment for a single nanoparticle qualitatively, using the Stoner-Wohlfarth model [9]. Next, we investigate the mean magnetic moment of a large ensemble of immobilized nanoparticles with parallel-aligned easy axes exposed to a sinusoidal excitation field quantitatively in

\section{*m.hofmann@uke.de}

Published by the American Physical Society under the terms of the Creative Commons Attribution 4.0 International license. Further distribution of this work must maintain attribution to the author(s) and the published article's title, journal citation, and DOI. a simulation study. In this setting, we find a good distinguishability of the response signals with respect to the easy-axis orientation. Application-wise, we use these findings to perform a data-driven estimation method for the spatial orientation of the easy axis of magnetic nanoparticles and provide a proof of principle in a magneticparticle-imaging $[10,11]$ setting where the orientation can be estimated tomographically. In this context, the proposed method provides an additional image contrast, such as the recently introduced temperature [12], viscosity [13,14], and core-size [15] contrasts, all of which are provided in addition to the spatial nanoparticle distribution. To this end, the proposed method allows us to simultaneously estimate the orientation and position of multiple objects labeled by immobilized nanoparticles with parallel-aligned easy axes.

Magnetization dynamics of a single immobilized nanoparticle.- In the general case of ferrofluids, two dynamic mechanisms influence the magnetization behavior of individual nanoparticles, Brownian and Néel rotational dynamics [5,6] (for recent reviews, see Refs. [1618]). Unlike ferrofluids, for immobilized nanoparticles, Brownian rotation is suppressed and only the Néel rotation derived from the phenomenological Landau-LifshitzGilbert equation remains [19,20].

Assuming a single domain structure (e.g., for nanoparticles with ferromagnetic cores with sufficiently small diameters [21]), neglecting particle-particle interactions, including an uniaxial anisotropy with easy axis $\hat{\mathbf{n}} \in S^{2}$ (Stoner-Wohlfarth model [9]), and including thermal noise, the magnetic moment $\mathbf{m}$ of an individual particle 
can be described by the Langevin equation with respect to its orientation $\hat{\mathbf{m}}:(0, T) \rightarrow S^{2}$ :

$$
\dot{\hat{\mathbf{m}}}=\tilde{\gamma}\left\{\left(\mathbf{B}_{\mathrm{eff}}+\tilde{D} \boldsymbol{\Gamma}\right) \times \hat{\mathbf{m}}+\alpha^{\prime}\left[\hat{\mathbf{m}} \times\left(\mathbf{B}_{\mathrm{eff}}+\tilde{D} \boldsymbol{\Gamma}\right)\right] \times \hat{\mathbf{m}}\right\},
$$

where $\mathbf{B}_{\text {eff }}=\mu_{0} \mathbf{H}+2\left(K_{\text {anis }} / M_{S}\right)(\hat{\mathbf{m}} \cdot \hat{\mathbf{n}}) \hat{\mathbf{n}}$ (with anisotropy constant $K_{\text {anis }}$ and saturation magnetization $M_{S}$ ), $\tilde{\gamma}=$ $\left(\gamma / 1+\alpha^{\prime 2}\right)$, in which $\gamma>0$ is the gyromagnetic ratio and $\alpha^{\prime}>0$ is the damping parameter, $\tilde{D}=$ $\sqrt{2\left(\alpha^{\prime} k_{B} T_{B} / \tilde{\gamma}\left(1+\alpha^{\prime 2}\right) m_{0}\right)}>0$ is the noise coefficient (with Boltzmann constant $k_{B}$, temperature $T_{B}$, and $m_{0}$ is the absolute value of the magnetic moment of the particle), and $\boldsymbol{\Gamma}$ is a white-noise component with $\left\langle\boldsymbol{\Gamma}_{i}(t)\right\rangle=$ $0,\left\langle\boldsymbol{\Gamma}_{i}\left(t_{1}\right) \boldsymbol{\Gamma}_{j}\left(t_{2}\right)\right\rangle=\delta_{i j} \delta\left(t_{1}-t_{2}\right)$, for all $t, t_{1}, t_{2}>0$, and $i, j=x, y, z$. The right-hand side of Eq. (1) can be split into three components with respect to their dependence on $\mathbf{H}, \hat{\mathbf{n}}$, or $\boldsymbol{\Gamma}$. The qualitative behavior of the magnetic moment of the particle strongly depends on the interplay between these three quantities. Here, we assume that the influence of thermal noise $\Gamma$ is on a low-to-moderate relative level such that the deterministic contributions of $\hat{\mathbf{n}}$ and $\mathbf{H}$ provide the main contributions to the behavior of the moment.

In a ferrofluid, the easy axis $\hat{\mathbf{n}}$ would dynamically change depending on the applied magnetic field. In contrast, $\hat{\mathbf{n}}$ is static for immobilized nanoparticles. In this case, the interplay between $\hat{\mathbf{n}}$ and $\mathbf{H}$ becomes apparent if the particle is subject to a magnetic sinusoidal excitation field in the $x$ direction (denoted by the unit vector $\mathbf{e}_{x} ; \mathbf{e}_{y}$ and $\mathbf{e}_{z}$ defined analogously). If $\hat{\mathbf{n}}$ is parallel to $\mathbf{e}_{x}$, the model given in Eq. (1) predicts that the main contribution of the magnetization response will be parallel to the excitation direction, which is qualitatively very similar to the response of mobile nanoparticles. However, if the easy axis $\hat{\mathbf{n}}$ is rotated out of the $x$ direction, the model given in Eq. (1) not only predicts response changes in the $x$ direction but significant signal contributions in the $y-z$ plane as well.

Magnetization dynamics of an ensemble of nanoparticles.-We proceed with a large ensemble of immobilized nanoparticles with parallel-aligned magnetic easy axes, as sketched in Fig. 1(a). Considering a sinusoidal excitation along $\mathbf{e}_{x}$ in relation to the magnetic easy-axis orientation $\hat{\mathbf{n}}(\alpha)=[\cos (\alpha), \sin (\alpha), 0]^{T}$, specified by the angle $\alpha \in$ $[0, \pi)$ between the excitation direction and the direction of the magnetic easy axis, the mean magnetic moment $\overline{\mathbf{m}}_{\alpha}$ of the ensemble can be simulated computationally via the Fokker-Planck equation approach, as described in Refs. [22-24]. The magnetization signal of such an ensemble is often picked up by induction coils. In this case, the coil signal is proportional to a projection of $\dot{\overline{\mathbf{m}}}_{\alpha}$. To this end, $\dot{\overline{\mathbf{m}}}_{\alpha} \cdot \mathbf{e}_{x}$ and $\dot{\overline{\mathbf{m}}}_{\alpha} \cdot \mathbf{e}_{y}$ are illustrated in Fig. 1(b) (a) SPIOs in

SPIOs in

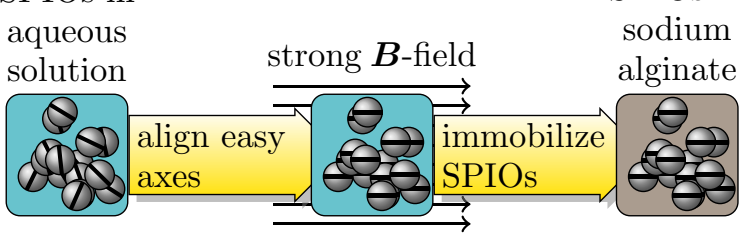

(b) $y$ excitation
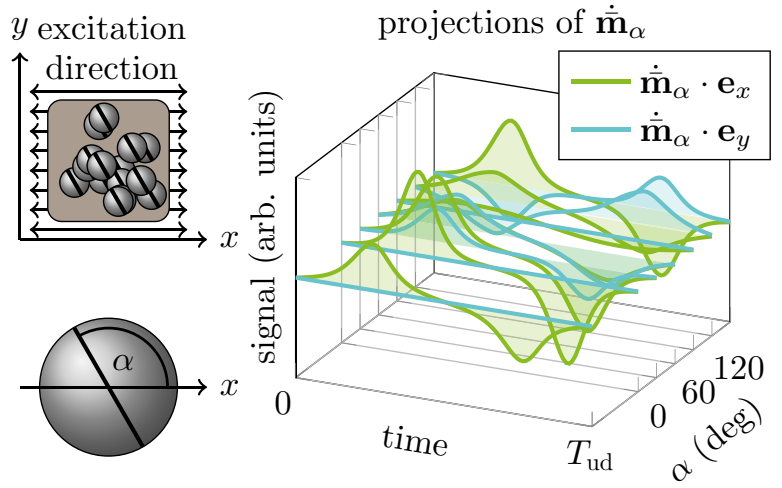

(c)

sample with
unknown
orientation $\beta$

samples with known

orientation $\beta$

orientation $\alpha$

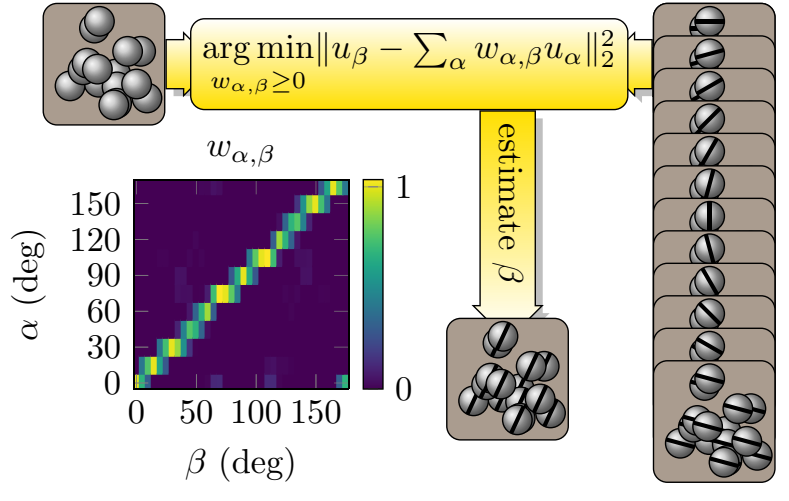

FIG. 1. An illustration of the basic idea to estimate the spatial orientation of the easy axis of immobilized magnetic nanoparticles (MNPs) with parallel-aligned magnetic easy axes. (a) Nanoparticles in aqueous solution are brought into a strong magnetic field to align their magnetic easy axis in parallel to the field. (b) Particle rotation is then rendered impossible by particle immobilization. A sinusoidal magnetic excitation field in the $x$ direction causes a magnetization response, which strongly depends on the angle $\alpha$ between the excitation direction and the direction of the magnetic easy axes. This is illustrated by the time signals $\dot{\overline{\mathbf{m}}}_{\alpha} \cdot \mathbf{e}_{x}$ and $\dot{\overline{\mathbf{m}}}_{\alpha} \cdot \mathbf{e}_{y}$, which are proportional to the signal that inductive $x$ and $y$ coils would pick up, respectively. On the contrary, no orientation dependency is observed for particles with a uniform easy-axis distribution (ud). (c) Next, concatenated time signals from $x$ and $y$ coils are considered. Let $u_{\alpha}$ be calibration signals of samples with known orientation and let $u_{\beta}$ be a signal of an unknown axis orientation, which we aim to estimate. Using a least-squares approach to approximate $u_{\beta}$ by a linear combination of the calibration signals $u_{\alpha}$ using non-negative weights $W_{\beta}=\left(w_{\alpha, \beta}\right)_{\alpha}$, one observes large weights whenever $\alpha$ and $\beta$ are close. In practice, this observation can be used as the basis for a data-driven estimator for the unknown orientation $\beta$. 
in relation to the easy-axis orientation $\alpha$ and for nonaligned nanoparticles as reference. The distinctiveness of the signals indicates that they contain information about the spatial orientation of the easy axis $\hat{\mathbf{n}}$ with respect to the direction of the magnetic excitation $\mathbf{e}_{x}$. To further investigate the distinguishability, we concatenate $\dot{\overline{\mathbf{m}}}_{\alpha} \cdot \mathbf{e}_{x}$ and $\dot{\overline{\mathbf{m}}}_{\alpha} \cdot \mathbf{e}_{y}$ into a single signal vector $u_{\alpha}$. Considering signals $u_{\beta}$ with unknown orientations $\beta$, we use a calibrationbased approach and try to approximate them by a linear combination of a set of calibration signals $u_{\alpha}$ using nonnegative weights, as illustrated in Fig. 1(c). The structure in the resulting weights highlights the distinguishability of the signals with respect to the easy-axis orientation and simultaneously can be used for estimation of $\beta$ in a data-driven approach.

Tomographic orientation estimation with magnetic particle imaging.- Until now, we have considered a setting where all particles will have the same parallel easyaxis orientation and are subject to the exact same magnetic excitation. Next, we consider the spatial distribution of immobilized magnetic nanoparticles with a spatially dependent particle distribution and orientation of the parallel-aligned easy axes, with the aim of obtaining a tomographic estimate of both. To this end, we move to a magnetic-particle-imaging (MPI) setting, where a static magnetic gradient field is superimposed with an excitation field leading to a spatially dependent magnetization response, used to tomographically image the nanoparticle distribution [10] along other image contrasts [11-15].

Experiments are performed with a preclinical MPI system (Bruker, Ettlingen, Germany) operating at a gradient strength of $-0.96,-0.98$, and $1.94 \mathrm{~T} \mu_{0}^{-1} \mathrm{~m}^{-1}$ in the $x$, $y$, and $z$ directions and a two-dimensional (2D) excitation field, generated by two orthogonal sinusoidal fields with slightly different frequencies and amplitudes of 12.59 and $13.55 \mathrm{mT} \mu_{0}^{-1}$ in the $x$ and $y$ directions, respectively, where $\mu_{0}$ is the vacuum permeability. This results in a field of view of size $26 \times 28 \mathrm{~mm}^{2}$ and a measurement repetition time of $652.8 \mu \mathrm{s}$. For signal reception, three orthogonal pickup coils aligned in the $x, y$, and $z$ directions are used. Each measurement vector $u$ is a concatenation of the three Fourier-domain induction signals sampled at a rate of 2.5 $\mathrm{MHz}$, one for each pickup coil.

A dot phantom, as sketched in Fig. 2, is printed using a Form 3 3D printer and clear resin (Formlabs Inc., Somerville, USA). The top part is a $8 \times 8 \times 4 \mathrm{~mm}^{3}$ cuboid with a cylindrical cavity at the top, with a diameter of 3 $\mathrm{mm}$ and a height of $3 \mathrm{~mm}$, fit to hold immobilized particles. It has a pin at the bottom to allow insertion into a series of connectors, each of which can be attached to a robot arm, which can be used to position the phantom in the MPI scanner. Each connector provides a defined phantom orientation inside the $x-y$ plane of the MPI system. The orientation is specified by the angle between the (a)

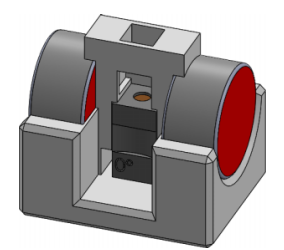

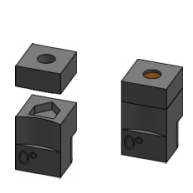

(b)

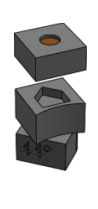

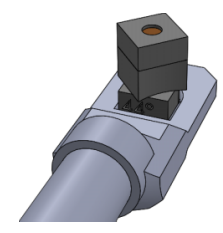

FIG. 2. A schematic of magnetic nanoparticle immobilization, parallel magnetic easy-axis alignment (a), and phantom orientation inside the MPI system (b). The phantom parts (dark gray) are assembled and the cylindrical depression is filled with a alginatetracer mixture (brown), which is quickly placed in a magnetic field (axial between red-sided magnets) to dry. The top part of the phantom can then be reattached to different connectors, each providing a defined easy-axis orientation inside the MPI system, once connected to the robot arm (blue and gray).

parallel-aligned magnetic easy axis and the $y$ axis of the MPI system. For the magnetic nanoparticles, perimag (Micromod Partikeltechnologie GmbH, Rostock, Germany), with an iron concentration of $89 \mathrm{mmol}_{\mathrm{Fe}} \mathrm{l}^{-1}$, is used. An amount of $20 \mu \mathrm{l}$ of the fluidal-particle suspension are mixed with about $21 \mu \mathrm{l}$ of sodium alginate powder inside the cavity and put in between two cylindrical neodymium magnets, creating a strong axial field measured by a model 460 three-channel gaussmeter with a three-axis high-sensitivity Hall probe (Lake Shore, Westerville, USA) to be $0.4 \mathrm{~T} \mu_{0}^{-1}$. Inside this field, the magnetic easy axes of the magnetic nanoparticles align in parallel to the external field by particle rotation $[7,8]$. Drying the mixture for $20 \mathrm{~min}$ immobilizes the magnetic nanoparticles and fixes the orientation of their easy axes permanently.

The relation between the measured signal $u_{\alpha}$ and the spatial distribution of the particles, described by the particle concentration vector $c_{\alpha}$, is linear and can be described by $S_{\alpha} c_{\alpha}=u_{\alpha}$, where $S_{\alpha}$ is the system matrix. Here, the subscript $\alpha$ labels the orientation of the easy axis of the particle, i.e., the angle between the parallel-aligned magnetic easy axis and the $y$ axis of the MPI system. Twelve different system matrices $S_{\alpha}$ are obtained using different easy-axis orientations, specified by the angles $\alpha$ in Table I. Each system matrix is measured in a $33 \times 33 \mathrm{~mm}^{2}$ field of view at $N=11 \times 11=121$ equidistant measurement positions. Additional measurements $u_{\beta, i}$ are taken for each of the 36 alignments $\beta$ listed in Table I and 21 phantom positions $i$ listed in the open-source software repository associated with this project [25]. For both calibration and the additional measurements, the signal-to-noise ratio is increased by block averaging 5000 consecutive MPI measurement cycles, corresponding to a total measurement time of about $3.3 \mathrm{~s}$ per position and angle.

For reconstruction, the multicontrast method introduced in Ref. [11] is applied, where all 12 system matrices are 
TABLE I. The magnetic easy-axis orientations used during calibration $(\alpha)$ and measurement $(\beta)$.

\begin{tabular}{lccccccc}
\hline \hline $\begin{array}{l}\alpha \\
\text { (degrees) }\end{array}$ & $\begin{array}{c}\alpha \\
\text { (degrees) }\end{array}$ & $\begin{array}{c}\beta \\
\text { (degrees) }\end{array}$ & $\begin{array}{c}\beta \\
\text { (degrees) }\end{array}$ & $\begin{array}{c}\beta \\
\text { (degrees) }\end{array}$ & $\begin{array}{c}\beta \\
\text { (degrees) }\end{array}$ & $\begin{array}{c}\beta \\
\text { (degrees) }\end{array}$ & $\begin{array}{c}\beta \\
\text { (degrees) }\end{array}$ \\
\hline 0 & 90 & 24 & 61 & 82 & 104 & 135 & 169 \\
15 & 105 & 25 & 62 & 83 & 105 & 136 & 170 \\
30 & 120 & 26 & 63 & 84 & 106 & 137 & 171 \\
45 & 135 & 29 & 66 & 87 & 109 & 140 & 174 \\
60 & 150 & 34 & 71 & 92 & 114 & 145 & 179 \\
75 & 165 & 44 & 81 & 102 & 124 & 155 & 9 \\
\hline \hline
\end{tabular}

used to obtain a 12-channel MPI tomogram, each channel $c_{\alpha}$ corresponding to one of the angles $\alpha$ in Table I. That is, for each measurement vector $u_{\beta, i}$ the Tikhonov regularized optimization problem

$$
\underset{\tilde{c}_{0^{\circ}, \ldots, \tilde{c}_{165^{\circ}} \in \mathbb{R}_{+}^{N}}^{\operatorname{argmin}}}{\arg }\left\|\left(\begin{array}{c}
\tilde{c}_{0^{\circ}} \\
\vdots \\
\tilde{c}_{165^{\circ}}
\end{array}\right)-u_{\beta, i}\right\|_{2}^{2}+\lambda\left\|\left(\begin{array}{c}
\tilde{c}_{0^{\circ}} \\
\vdots \\
\tilde{c}_{165^{\circ}}
\end{array}\right)\right\|_{2}^{2}
$$

is solved, where $S=\left(\begin{array}{lll}S_{0^{\circ}} & \cdots & S_{165^{\circ}}\end{array}\right)$ is the multicontrast system matrix. To this end, we use an open-source MPI reconstruction framework [26], a relative Tikhonov regularization parameter of $\lambda=0.01\left(\|S\|_{F}^{2} / N\right)$, and 5000

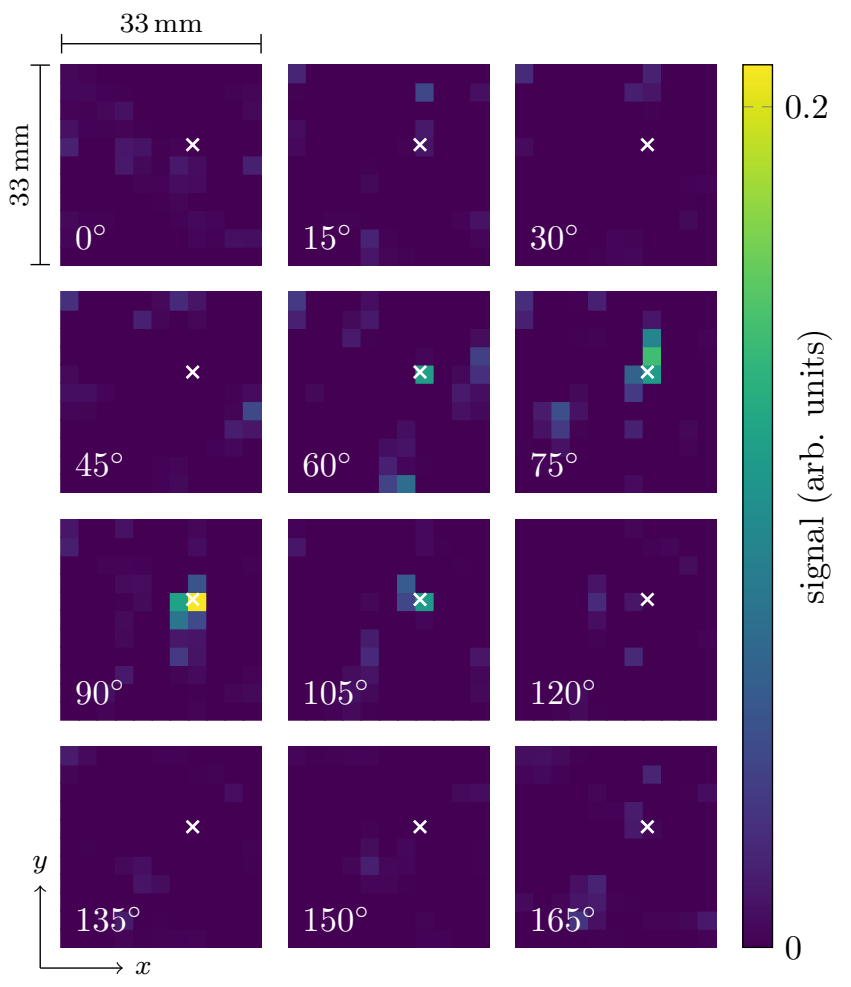

FIG. 3. A typical multicontrast tomogram $\left(\beta=82^{\circ}\right.$ and $i=$ $17)$ is shown. Each image shows a single channel with the corresponding label $\alpha$ at the bottom left corner. The largest signal contributions can be found in the channels $75^{\circ}$ and $90^{\circ}$ close to the phantom position (white marker). iterations of the iterative Kaczmarz solver, where $\|\cdot\|_{F}$ denotes the Frobenius norm. The noise background is reduced by selecting frequencies above $80 \mathrm{kHz}$ and a signal-to-noise ratio greater equal than 5 [27].

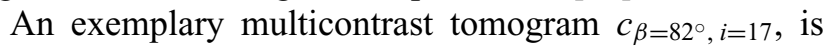
shown in Fig. 3, where the indices $\beta$ and $i$ label the alignment and position of the corresponding phantom, respectively. If we consider a single voxel $r$ and channel $\alpha$ inside the tomogram we obtain the non-negative numbers $c_{\alpha, r, \beta, i}$. Due to image noise, the $c_{\alpha, r, \beta, i}$ show a certain amount of spatial variation, which can be reduced by summing these numbers in a neighborhood $U(i)$, i.e., a radius of $4 \mathrm{~mm}$ around the respective position of the phantom, resulting in the weights $w_{\alpha, \beta, i}=\sum_{r \in U(i)} c_{\alpha, r, \beta, i}$ for each tomogram $c_{\beta, i}$, which are equivalent to the weights described in Fig. 1.

Similar to the observations made with the simulation setup, the individual weights $w_{\alpha, \beta, i}$ are large, where $\alpha$ and $\beta$ are close, which motivates the data-driven definition of the following alignment estimator. Let $f\left(\alpha ; b, c, \beta_{e}\right)=$ $b\left\{\cos ^{2}\left[\pi\left(\alpha-\beta_{e}\right)\right]\right\}^{c}$ be a $\pi$-periodic function with maximum at $\alpha=\beta_{e}$ and let

$$
\left(c_{\beta, i}^{*}, b_{\beta, i}^{*}, \beta_{\beta, i}^{*}\right)=\underset{c, b>0, \beta_{e} \in[0, \pi)}{\arg \min }\left\|\sum_{\alpha} w_{\alpha, \beta, i}-f\left(\alpha ; b, c, \beta_{e}\right)\right\|_{2}^{2}
$$

be the least-squares fit of this function to these weights. Then $\beta_{\beta, i}^{*}$ defines our estimator for the easy-axis alignment obtained from $c_{\beta, i}$.

Results.-Regardless of the phantom orientation $\beta$ and position $i$, the phantom can be located visually inside the MPI multicontrast tomograms. The largest signal contribution is found in pixels at or directly adjacent to the position of the phantom in channels $\alpha$ close to the alignment of the sample $\beta$, as shown, for example, in Fig. 3. In agreement with this observation, we find the weights $w_{\alpha, \beta, i}$ obtained from each tomogram to be large whenever $|\alpha-\beta|<30^{\circ}$, similar to the observations made in the simulation setup. Estimates for the alignment are successfully obtained from all MPI tomograms. The estimation errors have a mean of $0.3^{\circ}$ and a standard deviation of $2.5^{\circ}$. A closer analysis shows no spatial dependence of the estimation error. 
Conclusion and perspectives. - In this work, we show that the magnetization response of immobilized magnetic nanoparticles with parallel-aligned easy axes to an external magnetic excitation field can be used to estimate the angle between excitation and easy-axis direction. Our qualitative investigations of a single moment inside a one-dimensional magnetic excitation field predicts a nonzero magnetization response orthogonal to the excitation direction for easy-axis alignments nonparallel to the excitation direction. A quantitative simulation study of a large ensemble of nanoparticles shows distinct magnetization-signal changes with the variation of the angle between the excitation and the easy axis - a feature that can be attributed to the easyaxis alignment, since no such changes are observed for immobilized magnetic nanoparticles with uniformly distributed easy axes. A numerical analysis of the signals shows that a simple data-driven approach can be used for signal discrimination, as well as for the estimation of the angle between the excitation and the easy axis from a given signal.

Application-wise, we provide a proof of concept that the orientation of the easy axis and the spatial position of an ensemble of immobilized magnetic nanoparticles with parallel-aligned easy axes can be estimated in a MPI system. In this scenario, orientation estimation supplements submillimeter-accurate spatial localization [28] to provide additional information about the orientation, especially if its size is well below the current spatial resolution of the MPI, of around $1 \mathrm{~mm}$ [29], where image-processingbased approaches fail. In this study, a systematic error of $0.3^{\circ}$ and a larger standard error of $2.5^{\circ}$ are observed, which, combined, are well below the discretization step size of $15^{\circ}$ used for the calibration measurements, which are required for the data-driven approach used. Part of this error is likely to be caused by the signal-to-noise ratio, as found in a different multicontrast MPI setting [14]. A potential lever to address this issue consists of tailored nanoparticles with a large fraction of Néel relaxation and large uniaxial anisotropy as opposed to the ones used in this work, which lose much of their signal strength when immobilized. Although we restrict our setup to estimate orientations within a 2D plane only, the proposed method is generalizable to a $3 \mathrm{D}$ setup.

Orientation information can be invaluable for the navigation of microrobots in viscous environments. To this end, our findings could open up additional routes for the navigation of magnetically actuated microrobots. In the context of MPI, identical helical micromachines can be spatially selective actuated [30]. Moreover, alternating imaging and actuation of helical micromachines [31,32], magnetic microbeads [33], and swarming magnetite nanoparticles [34] have recently been shown. Since our proof of concept does not require a specialized imaging sequence, but only labeling with immobilized easy-axis-aligned nanoparticles, it would be feasible to obtain the additional orientation information in such a setting.

Acknowledgments.-T. Knopp gratefully acknowledges the financial support by the Federal Ministry of Education and Research (BMBF), under Grants No. 05M16GKA and No. 13XP5060B, and the State Ministry for Science and Research (BWFG, Behörde für Wissenschaft, Forschung und Gleichstellung), Hamburg (ahoi.digital project, Adaptive crossmodale Sensordatenerfassung). T. Kluth acknowledges funding by the German Research Foundation (DFG, Deutsche Forschungsgemeinschaft) under Project No. 426078691.

[1] A.-H. Lu, E. L. Salabas, and F. Schüth, Magnetic nanoparticles: Synthesis, protection, functionalization, and application, Angew. Chem. Int. Ed. 46, 1222 (2007).

[2] K. M. Krishnan, Fundamentals and Applications of Magnetic Materials (Oxford University Press, Oxford, 2016).

[3] S. Gul, S. B. Khan, I. U. Rehman, M. A. Khan, and M. Khan, A comprehensive review of magnetic nanomaterials modern day theranostics, Front. Mater. 6, 179 (2019).

[4] K. Wu, D. Su, J. Liu, R. Saha, and J.-P. Wang, Magnetic nanoparticles in nanomedicine: A review of recent advances, Nanotechnology 30, 502003 (2019).

[5] W. T. Coffey, P. J. Cregg, and Y. U. P. Kalmykov, in Advances in Chemical Physics (John Wiley \& Sons, Inc., 1992), p. 263.

[6] M. Shliomis and V. Stepanov, Theory of the dynamic susceptibility of magnetic fluids, Adv. Chem. Phys.: Relaxation Phenomena Condens. Matter 87, 1 (1994).

[7] T. Yoshida, Y. Matsugi, N. Tsujimura, T. Sasayama, K. Enpuku, T. Viereck, M. Schilling, and F. Ludwig, Effect of alignment of easy axes on dynamic magnetization of immobilized magnetic nanoparticles, J. Magn. Magn. Mater. 427, 162 (2017).

[8] A. L. Elrefai, T. Sasayama, T. Yoshida, and K. Enpuku, AC magnetization of immobilized magnetic nanoparticles with different degrees of parallel alignment of easy axes, IEEE Trans. Magn. 57, 1 (2020).

[9] E. C. Stoner and E. P. Wohlfarth, A mechanism of magnetic hysteresis in heterogeneous alloys, IEEE Trans. Magn. 27, 3475 (1991).

[10] B. Gleich and J. Weizenecker, Tomographic imaging using the nonlinear response of magnetic particles, Nature 435 , 1214 (2005).

[11] J. Rahmer, A. Halkola, B. Gleich, I. Schmale, and J. Borgert, First experimental evidence of the feasibility of multicolor magnetic particle imaging, Phys. Med. Biol. 60, 1775 (2015).

[12] C. Stehning, B. Gleich, and J. Rahmer, Simultaneous magnetic particle imaging (MPI) and temperature mapping using multi-color MPI, Int. J. Magn. Particle Imaging 2, ID 1612001, 6 (2016).

[13] M. Utkur, Y. Muslu, and E. U. Saritas, Relaxation-based viscosity mapping for magnetic particle imaging, Phys. Med. Biol. 62, 3422 (2017). 
[14] M. Möddel, C. Meins, J. Dieckhoff, and T. Knopp, Viscosity quantification using multi-contrast magnetic particle imaging, New J. Phys. 20, 083001 (2018).

[15] C. Shasha, E. Teeman, K. M. Krishnan, P. Szwargulski, T. Knopp, and M. Möddel, Discriminating nanoparticle core size using multi-contrast MPI, Phys. Med. Biol. 64, 074001 (2019).

[16] D. B. Reeves and J. B. Weaver, Approaches for modeling magnetic nanoparticle dynamics, Crit. Rev. Biomed. Eng. 42, 85 (2014).

[17] T. Kluth, Mathematical models for magnetic particle imaging, Inverse. Probl. 34, 083001 (2018).

[18] C. Shasha and K. M. Krishnan, Nonequilibrium dynamics of magnetic nanoparticles with applications in biomedicine, Adv. Mater. n/a, 1904131 (2020).

[19] L. D. Landau and E. M. Lifshitz, On the theory of the dispersion of magnetic permeability in ferromagnetic bodies, Phys. Z. Sowjetunion 5, 153 (1935).

[20] T. Gilbert, A Lagrangian formulation of the gyromagnetic equation of the magnetization field, Phys. Rev. 100, 1243 (1955).

[21] W. T. Coffey and Y. P. Kalmykov, Thermal fluctuations of magnetic nanoparticles: Fifty years after Brown, J. Appl. Phys. 112, 121301 (2012).

[22] T. Kluth, P. Szwargulski, and T. Knopp, Towards accurate modeling of the multidimensional magnetic particle imaging physics, New J. Phys. 21, 103032 (2019).

[23] H. Albers, T. Kluth, and T. Knopp, in 10th International Workshop on Magnetic Particle Imaging 2020, edited by T. Knopp and T. M. Buzug (Infinite Science Publishing, 2020), p. 2.

[24] H. Albers, T. Kluth, and T. Knopp, Simulating magnetization dynamics of large ensembles of single domain nanoparticles: Numerical study of Brown/Néel dynamics and parameter identification problems in magnetic particle imaging, Journal of Magnetism and Magnetic Materials, accepted for publication (2021).

[25] M. Möddel, F. Griese, T. Kluth, and T. Knopp, EasyAxisOrientationEstimation, https:/github.com/IBIResearch/ EasyAxisOrientationEstimation (2021), version: v1.0.0.
[26] T. Knopp, P. Szwargulski, F. Griese, M. Grosser, M. Boberg, and M. Möddel, MPIReco.jl: JULIA package for image reconstruction in MPI, Int. J. Magn. Part. Imag. (2019).

[27] K. Them, M. G. Kaul, C. Jung, M. Hofmann, T. Mummert, F. Werner, and T. Knopp, Sensitivity enhancement in magnetic particle imaging by background subtraction, IEEE Trans. Med. Imaging 35, 893 (2016).

[28] F. Griese, T. Knopp, R. Werner, A. Schlaefer, and M. Möddel, Submillimeter-accurate marker localization within low gradient magnetic particle imaging tomograms, Int. J. Magn. Particle Imaging 3 (2017).

[29] Z. W. Tay, P. W. Goodwill, D. W. Hensley, L. A. Taylor, B. Zheng, and S. M. Conolly, A high-throughput, arbitrary-waveform, MPI spectrometer and relaxometer for comprehensive magnetic particle optimization and characterization, Sci. Rep. 6, 34180 (2016).

[30] J. Rahmer, C. Stehning, and B. Gleich, Spatially selective remote magnetic actuation of identical helical micromachines, Sci. Robot. 2, eaal2845 (2017).

[31] A. C. Bakenecker, A. von Gladiss, T. Friedrich, U. Heinen, H. Lehr, K. Lüdtke-Buzug, and T. M. Buzug, Actuation and visualization of a magnetically coated swimmer with magnetic particle imaging, J. Magn. Magn. Mater. 473, 495 (2019).

[32] A. C. Bakenecker, A. von Gladiss, H. Schwenke, A. Behrends, T. Friedrich, K. Lüdtke-Buzug, A. Neumann, J. Barkhausen, F. Wegner, and T. M. Buzug, Navigation of a magnetic micro-robot through a cerebral aneurysm phantom with magnetic particle imaging, Sci. Rep. 11, 1 (2021).

[33] F. Griese, T. Knopp, C. Gruettner, F. Thieben, K. Müller, S. Loges, P. Ludewig, and N. Gdaniec, Simultaneous magnetic particle imaging and navigation of large superparamagnetic nanoparticles in bifurcation flow experiments, J. Magn. Magn. Mater. 498, 166206 (2020).

[34] K. Bente, A. C. Bakenecker, A. von Gladiss, F. Bachmann, A. Cébers, T. M. Buzug, and D. Faivre, Selective actuation and tomographic imaging of swarming magnetite nanoparticles, ACS Appl. Nano Mater. 4, 6752 (2021). 